\title{
Multiple Dynamic Quality Systems Using Hybrid Neuro-Ants Technique
}

\author{
Hsu-Hwa Chang ${ }^{1, *}$ Chih-Hsien Chen ${ }^{2}$ Ching-Shih Tsou ${ }^{1}$ \\ ${ }^{1}$ National Taipei College of Business, Taiwan \\ ${ }^{2}$ Lee-Ming Institute of Technology, Taiwan
}

\begin{abstract}
Robust parameter design has been successfully applied to a variety of engineering problems for enhancing the robustness of the system; however, it cannot deal with multiple dynamic quality systems. Although several other approaches have been presented to resolve this problem, they are unable to efficiently treat the situations that the control factors have continuous values. This study incorporates desirability functions into a hybrid neuro-ants technique to optimize the parameter design of multiple dynamic quality systems with continuous parameters. The objective is to find the best parameter settings so as to maximize simultaneously the robustness of each response. The proposed approach is illustrated with a constructed example.
\end{abstract}

Keywords: Artificial network networks, continuous ant colony optimization, dynamic systems, desirability functions, multiple responses, robust design.

\section{Introduction}

Parameter design is a critical phase in developing new products because it mostly determines the total production cost and quality. The robust design introduced by Taguchi is conventionally applied to optimize the parameter design problems, which uses orthogonal array to arrange the experiments and employs signal-to-noise ratios (SNRs) to evaluate the performance of the response of each experimental run. Through the robust design, the optimal factor/level combination can be determined to simultaneously reduce the response variation and bring the mean close to the target value. Although the robust design method has wide applications in practice, it is hard to use in multiple responses problems, especially in dynamic systems (Maghsoodloo et al., 2004; Robinson et al., 2004; Zang et al., 2005). Recently, the parameter design problems containing multiple dynamic responses have increasingly received attentions. Several researchers have begun to study this problem, such as the literature by Tong et al.
(2004), Wu and Yeh (2005), and Wang and Tong (2005). Considering a dynamic with multi-response system, suppose that there are $r$ output responses $Y=\left(y_{1}, y_{2}, \ldots, y_{r}\right)$ which are determined by a set of control factor vector $\mathbf{X}=\left(x_{1}, x_{2}, \ldots, x_{p}\right)$ and by a set of signal levels $M=\left(M_{1}, M_{2}, \ldots, M_{s}\right)$. The dynamic multiresponse system can be defined as:

$y_{j k}=f_{j k}\left(M_{k}, \mathbf{X}\right)+e_{j k}$

for $j=1,2, \ldots, r ; k=1,2, \ldots, s$.

where $f_{j k}$ denotes the function between the control factors and the $j$ th response at the $k$ th level of signal factor; and $e_{j k}$ is a random error.

For each dynamic response, assumes that a linear form exists between the response and the signal factors. The ideal function can be expressed as $y=\beta M+e$, where $y$ denotes the response, $M$ stands for the signal factor, $\beta$ is the slope or system sensitivity, and $e$ represents the random error. Further, dynamic systems can be classified into dynamic nominal-the-better (DNB), dynamic larger-the-better (DLB), and dynamic smaller-the-better (DSB) according to the desired target slope. Hence, the ideal target function is replaced as $Y=\beta_{t} M+e$, where $\beta_{t}$ is the desired target slope. For the response type DNB, $\mathrm{DLB}$, and DSB, the value of the slope is $0<\beta_{t}<\infty$, $\beta_{t}=\infty$, and $\beta_{t}=0$, respectively.

Despite of several approaches are presented for resolving multiple dynamic systems, those methods can only obtain the discrete combinations among the specified control factor levels. In other words, they are unable to achieve the real optimal factor combination if the control factors have continuous values. In this work, we propose a novel optimization approach using desirability functions and a hybrid neuro-ants technique for resolving the multiple dynamic systems, the obtained parameter settings can be any value within their bounds. The hybrid neuroants integrates artificial neuro networks (ANNs) and continuous ant colony optimization (CACO) to model the system and to optimize the parameter design. 


\section{The Proposed Approach}

The proposed approach consists of three stages. First, experimental data are collected to train an ANN to represent the response function model of a dynamic multi-response system, $f_{j k}$, which is capable of predicting the corresponding responses by giving a specific factor combination. Second, the objective function of the system is formulated by using desirability functions to transform and integrate the predicted responses. Finally, a CACO algorithm is utilized to obtain the optimal objective value and the corresponding factor combination. Figure 2 shows the flowchart of the approach.

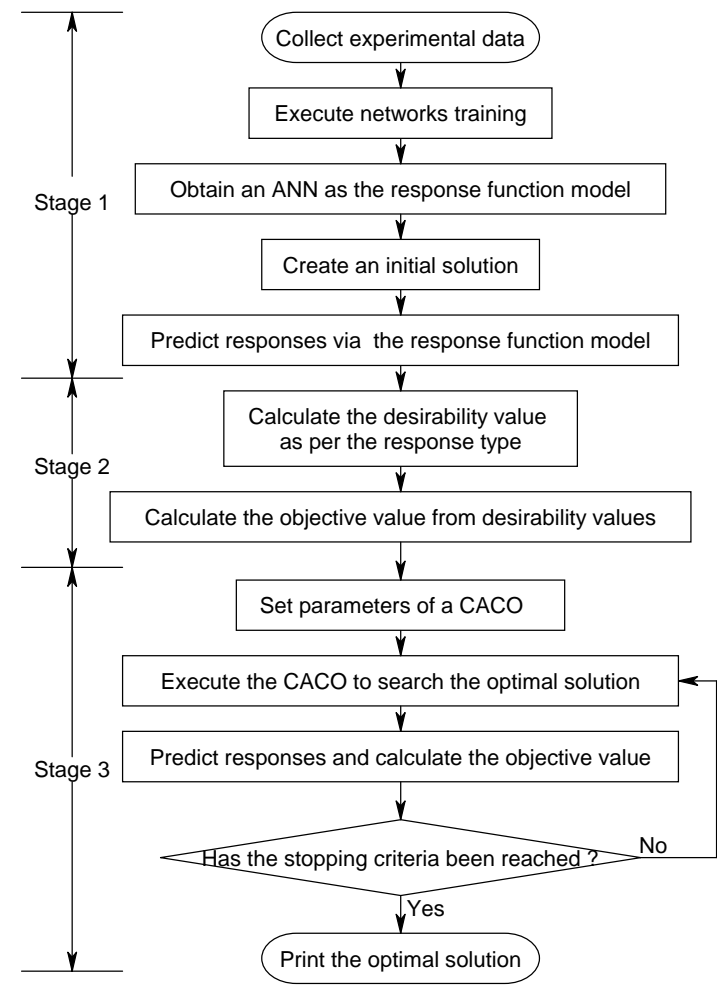

Figure 2. The flowchart of the proposed approach

\subsection{Response function modeling}

This stage uses an ANN to model the response function. The input and output data are assigned as the level values for the control factor, noise factor, and signal factor, and the responses, respectively. A welltrained neural network represents the system's response model, i.e., $\hat{y}_{j k}=f_{j k}\left(M_{k}, \mathbf{X}\right)$. For detailed discussion on how an ANN applied to parameter design, readers can refer to Rowlands et al. (1996), Chiu et al. (1997), and Su and Chang (2000). The processes of this stage are as follows:
Step 1. Collect the training and testing patterns for input and output layers from the experimental data.

Step 2. Select several ANN structures for training.

Step 3. Set learning rate, momentum coefficient and execution iterations $L$.

Step 4. For each network execute Steps $5-8 \mathrm{~L}$ times.

Step 5. Initialize randomly weights between layers.

Step 6. Apply the sigmoid function $f=1 /\left(1+e^{-x}\right)$ to predict the outputs.

Step 7. Calculate the error between the predicted output and the target output.

Step 8. Adjust the weights of the network.

Step 9. Choose the best one from the several trained networks as the system's response function model. The performance evaluation criterion for the network training is the root of mean-square error (RMSE).

\subsection{The objective function}

The desirability functions introduced by Harrington (1965) are modified to formulate the objective function of a multiple dynamic system. The desirability function transforms a predicted response to a scale-free value $d$, called desirability. It is a value between 0 and 1 , and it increases as the desirability of the corresponding response increases. For the three types of dynamic responses, the desirability value of the predicted response $\hat{y}_{j k}$ can be developed as follows:

- DNB: $d^{D N B}=\exp \left(-\left|Z^{D N B}\right|\right)$

where $Z^{D N B}=\frac{1}{s} \sum_{k=1}^{s} \frac{2 \hat{y}_{j k}-\left(y_{j k}^{\max }+y_{j k}^{\min }\right)}{y_{j k}^{\max }-y_{j k}^{\min }}$;

- DLB: $d^{D L B}=\exp \left(-\left(\exp \left(-Z^{D L B}\right)\right)\right)$ where $Z^{\text {DLB }}=\frac{1}{s} \sum_{k=1}^{s} \frac{\hat{y}_{j k}-y_{j k}^{\min }}{y_{j k}^{\min }}$

- DSB: $d^{D S B}=\exp \left(-\left(1+Z^{D S B}\right)\right)$

where $Z^{D S B}=\frac{1}{s} \sum_{k=1}^{s} \frac{\hat{y}_{j k}-y_{j k}^{\max }}{y_{j k}^{\max }}$.

For Eqs. (2)-(4), the bounds $y_{j k}^{\max }$ and $y_{j k}^{\min }$ represent the upper specification limit (USL) and lower specification limit (LSL) for the $j$ th response at the $k$ th signal level.

To enhance the overall performance of the multiple responses, we integrate the multiple desirability values into a single objective value:

$D=\sqrt[r]{\prod_{j=1}^{r} d_{j}}$

where $d_{j}$ denotes the desirability value for the $j$ th response; $j=1,2, \ldots, r$. 


\subsection{Optimizing by a CACO}

The CACO algorithms presented by Jayaraman et al. (2000) and Vijayakumar et al. (2003) are modified and implemented to our problem. A CACO utilizes bi-level procedures which include local and global searches. Local search ants select a local trail $i$ with a probability ${ }_{P_{i}}(t)=\tau_{i}(t) / \sum_{k} \tau_{k}(t)$, where $k$ is the number of trail solutions, $\tau_{i}(t)$ is the pheromone trail on solution $i$ at time $t$. After selecting the destination, the ant moves through a short distance $\Delta(t, R)=R\left(1-r^{(1-t / T) b}\right)$, where $R$ is maximum search radius, $r$ is a random number from [0,1], $T$ is the total number of iterations of the algorithm, and $b$ is a positive parameter controlling the degree of nonlinearity. A global search is done sequentially by crossover and mutation operations. The subsequent values of the variables of the child are set to the corresponding value of a randomly chosen parent with a crossover probability $\left(P_{c}\right)$. Mutation operation adds or subtracts a value to/from each variable with mutation probability $\left(P_{m}\right)$. The mutation step size is the same as the above distance $\Delta(t, R)$. Performing a CACO, ants are repeatedly sent to trail solutions in order to optimize the objective value. The total number of ants (denoted by $A$ ) is set as half the total number of trail solutions (denoted by $S$ ). The number of global ants (denoted by $G$ ) and the number of local ants (denoted by $L$ ) are set as $80 \%$ and $20 \%$ of the total number of ants, respectively.

Step 1. Set parameter values including: $S, A, \rho, \tau_{0}, P_{c}$, $P_{m}, T, b, R$, and bounds of each control factor.

Step 2. Create $S$ trail solutions. Estimate the outputs of the trail solutions through the ANN model.

Step 3. Calculate the objective values of the trail solutions

Step 4. Set the initial pheromone value of all trails.

Step 5. Repeat steps 6-9 until the stopping criteria has reached.

Step 6. Send $L$ ants to the selected trail solutions for local search.

Step 7. If the solution is improved, move the ants to the new solution and update the pheromone value.

Step 8. Send $G$ ants to global trails and generate their offspring by crossover and mutation.

Step 9. Evaporate pheromone for all trails.

\section{Illustrative Example}

A constructed example with multiple dynamic responses is studied. This system contains six responses, eight control factors, three signal levels, and two noise levels. The control factors $x_{1}, x_{2}, x_{3}, x_{4}$, $x_{5}, x_{6}, x_{7}$ and $x_{8}$ are allocated to orthogonal array $\mathrm{L}_{18}$ in order. Table 1 lists the types and bounds of all responses. The hypothetical experimental data obtained by the Monte Carlo simulation are given in Table 2.

Table 1. The specifications for the responses

\begin{tabular}{|c|c|c|c|c|c|c|c|c|}
\hline Response & $\mathrm{y}_{1}$ & \multicolumn{2}{|c|}{$\mathrm{y}_{2}$} & $\mathrm{y}_{3}$ & $\mathrm{y}_{4}$ & \multicolumn{2}{c|}{$\mathrm{y}_{5}$} & $\mathrm{y}_{6}$ \\
\hline Type & DLB & \multicolumn{2}{|c|}{ DNB } & DSB & DLB & \multicolumn{2}{c|}{ DNB } & DSB \\
\hline \multirow{2}{*}{ Bounds } & LSL & LSL & USL & USL & LSL & LSL & USL & USL \\
\cline { 2 - 10 } & $y_{j k}^{\min }$ & $y_{j k}^{\min }$ & $y_{j k}^{\max }$ & $y_{j k}^{\max }$ & $y_{j k}^{\min }$ & $y_{j k}^{\min }$ & $y_{j k}^{\max }$ & $y_{j k}^{\max }$ \\
\hline $\mathrm{M}_{1}=5$ & 35 & 14 & 26 & 650 & 175 & 1.75 & 3.25 & 130 \\
\hline $\mathrm{M}_{2}=10$ & 70 & 28 & 52 & 1300 & 350 & 3.5 & 6.5 & 260 \\
\hline $\mathrm{M}_{3}=15$ & 105 & 42 & 78 & 1950 & 525 & 5.25 & 9.75 & 390 \\
\hline
\end{tabular}

To train ANNs, we randomly select 520 training patterns and 128 testing patterns from Table 2 . Learning rate is set as between 0.01 and 0.3. The momentum coefficient is 0.85 . The number of iterations is 40,000. From several options of the trained networks, we select the structure 10-18-6 which has the smallest testing RMSE 0.0644. By using the ANN to predict the responses, the desirability values can be obtained as per their quality characteristics. The CACO algorithm is then applied to maximize the objection value in Eq. (5). The operational conditions of the CACO are $S=200, A=100$, $\rho=0.95, \tau_{0}=1, P_{c}=0.9, P_{m}=0.1, T=15$, and $b=10$. The bounds for control factors $x_{1}$ is $(1,2)$, for other factors are set as $(1,3)$. The CACO algorithm is executed over 20 runs to obtain the best settings $(1.2,1.4,2.6$, $1.1,3,2.2,1,2.9$ ), which has the largest $D$ value 0.8116 . The obtained settings are not restricted to the level values of the experiments. Table 3 lists the results of the implementation.

\section{Conclusion}

A novel approach incorporates desirability functions into a hybrid neuro-ants technique is proposed to optimize multiple dynamic systems and is implemented with an illustrative example. The proposed approach provides a generalization solution for parameter design and can be applied to diverse industrial fields. Through appropriate modification, the approach can be reduced to deal with most of the situations that practitioners may encounter, including static multiple responses, simple dynamic systems, and general static problems. 
Table 2. The responses of the system

\begin{tabular}{|c|c|c|c|c|c|c|c|c|c|c|c|c|c|c|c|c|c|c|c|}
\hline \multirow{3}{*}{ No } & \multirow{3}{*}{$\begin{array}{c}\text { Noise } \\
\text { factor } \\
\mathrm{s}\end{array}$} & \multicolumn{18}{|c|}{ Responses } \\
\hline & & \multicolumn{3}{|c|}{$\mathrm{y}_{1}$} & \multicolumn{3}{|c|}{$\mathrm{y}_{2}$} & \multicolumn{3}{|c|}{$\mathrm{y}_{3}$} & \multicolumn{3}{|c|}{$\mathrm{y}_{4}$} & \multicolumn{3}{|c|}{$\mathrm{y}_{5}$} & \multicolumn{3}{|c|}{$\mathrm{y}_{6}$} \\
\hline & & $\mathrm{M}_{1}$ & $\mathrm{M}_{2}$ & $\mathrm{M}_{3}$ & $\mathrm{M}_{1}$ & $\mathrm{M}_{2}$ & $\mathrm{M}_{3}$ & $\mathrm{M}_{1}$ & $\mathrm{M}_{2}$ & $\mathrm{M}_{3}$ & $\mathrm{M}_{1}$ & $\mathrm{M}_{2}$ & $\mathrm{M}_{3}$ & $\mathrm{M}_{1}$ & $\mathrm{M}_{2}$ & $\mathrm{M}_{3}$ & $\mathrm{M}_{1}$ & $\mathrm{M}_{2}$ & $\mathrm{M}_{3}$ \\
\hline \multirow{2}{*}{1} & $\mathrm{~N}_{1}$ & 54 & 98 & 171 & 23.8 & 49.0 & 45.3 & 451 & 1110 & 1198 & 204 & 543 & 872 & 1.4 & 4.3 & 6.8 & 111 & 226 & 321 \\
\hline & $\mathrm{N}_{2}$ & 40 & 107 & 142 & 12.8 & 39.7 & 53.2 & 504 & 1435 & 1055 & 223 & 488 & 893 & 2.6 & 4.0 & 2.8 & 118 & 205 & 270 \\
\hline \multirow{2}{*}{2} & $\mathrm{~N}_{1}$ & 49 & 80 & 127 & 19.9 & 34.7 & 55.7 & 742 & 962 & 1351 & 180 & 416 & 920 & 2.0 & 6.5 & 7.1 & 106 & 199 & 264 \\
\hline & $\mathrm{N}_{2}$ & 42 & 151 & 157 & 22.0 & 52.8 & 62.0 & 493 & 1176 & 1472 & 251 & 621 & 855 & 2.5 & 6.9 & 7.4 & 122 & 176 & 314 \\
\hline \multirow{2}{*}{3} & $\mathrm{~N}_{1}$ & 55 & 102 & 143 & 16.1 & 43.8 & 40.5 & 494 & 913 & 1351 & 188 & 564 & 901 & 3.3 & 6.7 & 10.0 & 104 & 206 & 321 \\
\hline & $\mathrm{N}_{2}$ & 49 & 93 & 119 & 15.4 & 37.3 & 50.5 & 580 & 990 & 1659 & 253 & 605 & 815 & 2.9 & 4.7 & 9.3 & 133 & 188 & 365 \\
\hline 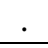 & \multicolumn{19}{|c|}{$\cdots \cdots$} \\
\hline \multirow{2}{*}{17} & $\mathrm{~N}_{1}$ & 45 & 91 & 193 & 15.1 & 52.6 & 70.7 & 553 & 910 & 1298 & 251 & 649 & 856 & 2.3 & 4.1 & 6.8 & 129 & 162 & 280 \\
\hline & $\mathrm{N}_{2}$ & 62 & 105 & 184 & 24.8 & 34.3 & 70.5 & 374 & 908 & 882 & 209 & 482 & 985 & 2.1 & 3.3 & 4.7 & 71 & 227 & 282 \\
\hline \multirow{2}{*}{18} & $\mathrm{~N}_{1}$ & 43 & 117 & 166 & 22.7 & 29.3 & 35.3 & 419 & 736 & 1497 & 323 & 478 & 719 & 1.7 & 5.8 & 10.5 & 99 & 233 & 261 \\
\hline & $\mathrm{N}_{2}$ & 44 & 127 & 194 & 24.7 & 53.0 & 69.4 & 458 & 836 & 1437 & 254 & 480 & 703 & 2.6 & 5.4 & 8.3 & 89 & 161 & 276 \\
\hline
\end{tabular}

Table 3. The results of implementing the proposed approach

\begin{tabular}{|r|c|c|c|c|c|c|c|c|c|c|c|c|c|c|}
\hline \multicolumn{1}{|c|}{ Control factor values } & \multicolumn{1}{c|}{$d$ values } & \multirow{2}{*}{$D$} \\
\cline { 1 - 10 } & \multicolumn{1}{|c|}{$x_{2}$} & $x_{3}$ & $x_{4}$ & $x_{5}$ & $x_{6}$ & $x_{7}$ & $x_{8}$ & $\mathrm{y}_{1}$ & $\mathrm{y}_{2}$ & $\mathrm{y}_{3}$ & $\mathrm{y}_{4}$ & $\mathrm{y}_{5}$ & $\mathrm{y}_{6}$ & \\
\hline 1.2 & 1.4 & 2.6 & 1.1 & 3 & 2.2 & 1 & 2.9 & 0.8081 & 0.9422 & 0.6864 & 0.9111 & 0.7101 & 0.8454 & 0.8116 \\
\hline
\end{tabular}

\section{References}

[1] Chiu, C. C., Su, C. T., Yang, G. H., Huang, J. S., Chen, S. C. and Cheng, N. T., "Selection of optimal parameters in gas-assisted injection moulding using a neural network model and the Taguchi method," International Journal of Quality Science, 2, 106-120, 1997.

[2] Jayaraman, V. K., Kulkarni, B. D., Karale, S. and Shelokar, P., "Ant colony framework for optimal design and scheduling of batch plants. Computers and Chemical Engineering,” 24, 1901-1912, 2000.

[3] Maghsoodloo, S., Ozdemir, G., Jordan, V. and Huang, C. H., "Strengths and limitations of Taguchi's contributions to quality, manufacturing, and process engineering," Journal of Manufacturing systems, 23(2), 73-126, 2004.

[4] Robinson, T. J., Borror, C. M. and Myers, R. H., "Robust parameter design: a review,” Quality and Reliability Engineering International, 20, 81-101, 2004.

[5] Rowlands, H., Packianather, M. S. and Oztemel, E., "Using artificial neural networks for experimental design in off-line quality,” Journal of Systems Engineering, 6, 46-59, 1996.
[6] Su, C. T. and Chang H. H., “Optimization of parameter design: an intelligent approach using neural network and simulated annealing," International Journal of Systems Science, 31(12), 1543-1549, 2000.

[7] Tong, L. I., Wang, C. H., Chen, C. C. and Chen, C. T., "Dynamic multiple responses by ideal function analysis,” European Journal of Operational Research, 156, 433-444, 2004.

[8] Vijayakumar, K., Prabhaharan, G., Asokan, P. and Saravanan, R., "Optimization of multi-pass turning operations using ant system," International Journal of Machine Tool \& Manufacture, 43, 1633-1639, 2003.

[9] Wang, C-H and Tong, L. I., "Optimization of dynamic multi-response problems using grey multiple attribute decision making," Quality Engineering, 17, 1-9, 2005.

[10] Wu, F. C. and Yeh, C. H., "Robust design of multiple dynamic quality characteristics,” International Journal of Advanced Manufacturing Technology, 25, 579-588, 2005.

[11] Zang, C., Friswell, M. I. and Mottershead, J. E., A review of robust optimal design and its application in dynamics, Computers and structures, 83, 315-326, 2005. 